\title{
De la génétique à la mimétique : mutations culturelles dans les publicités de voitures en Grande-Bretagne
}

\section{Marie-Hélène Fries-Verdeil}

\section{(2) OpenEdition}

\section{Journals}

Édition électronique

URL : http://journals.openedition.org/asp/4083

DOI : $10.4000 /$ asp.4083

ISSN : 2108-6354

Éditeur

Groupe d'étude et de recherche en anglais de spécialité

\section{Édition imprimée}

Date de publication : 1 décembre 1994

Pagination : 183-191

ISSN : 1246-8185

\section{Référence électronique}

Marie-Hélène Fries-Verdeil, « De la génétique à la mimétique : mutations culturelles dans les publicités de voitures en Grande-Bretagne », ASp [En ligne], 5-6 | 1994, mis en ligne le 12 décembre 2013,

consulté le 19 avril 2019. URL : http://journals.openedition.org/asp/4083 ; DOI : 10.4000/asp.4083

Ce document a été généré automatiquement le 19 avril 2019

Tous droits réservés 


\title{
De la génétique à la mimétique : mutations culturelles dans les publicités de voitures en Grande-Bretagne
}

\author{
Marie-Hélène Fries-Verdeil
}

1 Cette communication se propose d'analyser les liens entre science et culture populaire à partir d'un cas précis, celui des publicités automobiles. La publicité nous offre ici un champ d'investigation privilégié, au carrefour entre les caractéristiques techniques d'un produit donné, et tout le potentiel d'images, de rêves et de valeurs que ce même produit peut recéler. Mais pourquoi avoir choisi plus précisément l'automobile? Il s'est avéré, en partant d'un corpus de 380 publicités enregistrées sur la châne britannique ITV en août 1993, que les voitures étaient l'un des produits de grande consommation où le technique et le culturel se métissaient le plus, puisque les prouesses mécaniques allaient souvent de pair avec la création d'une image idéalisée du conducteur. «Dis-moi quelle voiture te tente, et je te dirai qui tu es ».

2 Le terme de «mutations culturelles » nous situe d'emblée au sein d'un paradoxe, puisqu'il s'agit d'éclairer un phénomène culturel à l'aide d'une analogie avec certaines lois de la nature, ici la théorie des gènes. Cette approche doit beaucoup au zoologiste britannique Richard Dawkins (Dawkins 1976). Pour Dawkins, les gènes se définissent, dans le monde du vivant, comme des unités minimales qui ont la faculté de se reproduire identiques à elles-mêmes. À partir de là, Dawkins fait une extrapolation en forgeant le néologisme de "mème", d'après le grec mimesis, pour désigner, au sein de la culture, des unités minimales qui partagent cette faculté de se reproduire avec fidélité (par exemple les airs de chanson, les styles de chaussure, et bien sûr les publicités.) Les gènes ont en outre la propriété de se transformer, par mutation, dans le but d'être mieux adaptés à leur environnement. Il s'agit maintenant d'analyser, comment les publicités de voitures, à partir d'un " gène culturel », ou mème originel, sont également sujettes à des mutations afin de mieux s'adapter à leur public cible. Une première mutation consiste en un changement du type de discours, qui glisse soit vers le sérieux scientifique, soit vers le 
burlesque, s'écartant du style publicitaire habituel. Une deuxième mutation permet de passer du domaine de l'information au monde des valeurs, de l'objet à sa signification, et fait de l'automobile un objet moderne valorisant pour le consommateur.

3 Le même originel se retrouve ici présenté dans deux versions différentes par les firmes Nissan et Ford ${ }^{1}$. La publicité pour Nissan se borne à énumérer, avec le bagout d'un camelot de foire, les caractéristiques techniques de la voiture et son prix. Pour la Ford Mondéo, c'est la sécurité qui prime. La voix «off» le démontre méthodiquement, en déclinant point par point tous les procédés techniques assurant une meilleure sécurité. Les images l'illustrent, fonctionnant en redondance par rapport au texte oralisé. Les angles de prise de vue le suggèrent métaphoriquement. Les gros plans sur la voiture alternent avec des panoramiques sur la nature et le ciel, depuis les nuages lourds d'orage de la première image, jusqu'au lever de soleil serein qui clôt la publicité.

Pourquoi identifier ces deux publicités comme contenant un gène culturel, un mème identique? C'est que d'une part elles remplissent la même fonction, faire vendre une voiture, comme on peut identifier un gène au fait qu'il donne des yeux bleus ou des petits pois ridés. Et d'autre part elles ont une structure commune, qui, pour n'être pas doublement hélicoïdale comme celle de l'ADN, n'en est pas moins très claire : le texte est centré sur les qualités conférées à la voiture par ses caractéristiques techniques, il est lu par une voix invisible qui s'adresse directement au public sur le mode de l'apostrophe. Les images montrent le véhicule sous tous ses angles, et démontrent visuellement ses atouts mécaniques.

5 À partir de ce mème de base, on peut repérer différentes mutations, qui, comme en génétique, ont pour but une meilleure adaptation à l'environnement, ici le public cible. La première mutation nette est d'ordre formel. La publicité automobile, tout en gardant apparente la structure du mème originel, cherche alors son inspiration dans d'autres types de discours, depuis le sérieux scientifique jusqu'au burlesque de la farce.

6 Le publi-documentaire intitulé «Focus on Ford» fait avec précision la preuve de la sécurité offerte par la Ford Mondeo, tout comme le mème originel, mais la démonstration s'effectue dans le style des émissions scientifiques de vulgarisation, Tomorrow's World par exemple pour la Grande-Bretagne ${ }^{2}$. La voix « off » se matérialise sous la forme d'un couple de présentateurs, la publicité acquiert un titre, Focus on..., qui pourrait être celui de n'importe quelle émission d'information, et les sujets à la troisième personne remplacent l'usage de l'apostrophe ${ }^{3}$. L'image se réduit alors à une illustration visuelle stricte de chaque étape de la démonstration, à l'aide de gros plans sur les différents éléments de la ceinture de sécurité, et sur le système de protection du conducteur par sac gonflable. Pourtant, l'omniprésence du logo de Ford, qui apparaît à la fois dans le titre, et en arrièreplan derrière chaque présentateur, définit clairement un cadre publicitaire, laissant au téléspectateur le soin de tirer ses propres conclusions.

7 La publicité pour la Vauxhall Astra présente une situation tout à fait similaire en apparence ${ }^{4}$. Un présentateur vante les mérites techniques du modèle, et ses arguments sont illustrés par une démonstration de déclenchement du sac gonflable de protection in situ. Le type de discours est néanmoins fort différent. Nous sommes ici dans le contexte d'un sketch, avec des personnages, un double public, une mise en scène qui habille la démonstration centrale d'un « avant » et d'un « après » burlesques. La parodie naît ici de la visualisation du jeu sur le mot "dummy», dont le signifiant unique renvoie à deux signifiés différents, mannequin et personnage niais. Elle se nourrit d'un de ces renversements de situation qui est le fondement même de la farce: normalement, un 
mannequin est fait pour remplacer un être humain, alors que c'est le contraire qui se produit ici : comme le mannequin nécessaire à la démonstration n'a pas pu être livré à temps, un employé apparemment un peu simplet s'offre à prendre sa place. Le mode parodique vient, semble-t-il, s'attaquer au présupposé communément admis selon lequel la sécurité est une affaire sérieuse. Pourtant, cette déviation se retourne en fait en démonstration, grâce à l'existence d'un double public: journalistes invités à la présentation d'un côté, et téléspectateurs de l'autre. L'élément de farce introduit une complicité entre le présentateur et le public cible, complicité dont les journalistes sont exclus. Eux n'y voient que du feu, mais les téléspectateurs savent l'excellent degré de sécurité offert par ce modèle Vauxhall, à tel point que le constructeur peut se payer le luxe de faire faire la démonstration du sac gonflable de protection par un homme en chair et en os.

8 Les exemples de Ford et de Vauxhall montrent clairement comment un même message publicitaire, "la voiture $\mathrm{X}$ ou $\mathrm{Y}$ offre les conditions de sécurité optimales», peut emprunter des formes de discours extérieures à la publicité, le reportage de vulgarisation scientifique ou la farce en l'occurrence. Ce changement formel tend bien sûr à renouveler l'intérêt du public en introduisant un élément de surprise, mais il ne remet pas en cause la structure du mème originel, qui montre pour démontrer. Le rapport entre un texte démonstratif et des images illustratives reste donc inchangé.

9 En revanche, la deuxième mutation repérée allie aux variations dans la forme du discours un changement de focalisation. L'accent n'est plus mis sur le produit (la voiture) mais sur le destinataire (l'acheteur potentiel). L'information cède alors le pas à la signification, pour mieux impliquer le destinataire. Cette création d'une image de soi dans laquelle l'acheteur potentiel peut se projeter peut aller du plus quotidien au plus romancé.

Dans la publicité pour Land Rover, la description orale des caractéristiques de la voiture a totalement disparu, laissant place à une voix «off» qui suit le fil des pensées de l'occupant de la voiture, sans chercher ni à faire un tri ni à créer de connexion logique ${ }^{5}$. Nous sommes très proches ici de la technique du «stream of consciousness » mise au point par Virginia Woolf 6 .

11 Nous nous trouvons en présence d'un texte fondé sur l'ellipse, qui juxtapose des remarques en omettant toute référence, ce qui crée un effet d'inattendu. Les images, en revanche, fournissent en contrepoint des références situationnelles qui permettent de reconstituer les silences elliptiques, et de pallier l'absence de contexte, sans rien ôter pour autant à la spontanéité d'une pensée prise sur le vif.

On voit donc clairement ici, avec ces publicités pour Rover, que le point de vue de l'annonceur, relayé par un présentateur qui vante les mérites du produit, a fait place à la voix du consommateur, ici les occupants de la 4/4. L'image de la voiture s'est diluée dans les paysages qu'elle traverse, alors que les automobilistes, au contraire, en nous laissant pénétrer dans leurs pensées, même brièvement, nous permettent d'esquisser un portrait d'eux, celui de jeunes cadres dynamiques aimant allier la stabilité et les joies de leur vie de famille (voix féminine) aux changements constants du monde dans lequel ils évoluent (voix masculine), et concilier l'attrait de l'aventure avec l'assurance d'un confort exceptionnel. La présentation de l'objet se trouve alors confiée aux images, qui démontrent visuellement les prouesses techniques de la Range Rover : la nervosité de sa reprise (même pour dépasser un long camion, ou, pire, un groupe de cyclistes), et sa rapidité de passage de la position basse (route) à la position haute (tous terrains). Le message final, «Range Rover, you never know where it will take you " est un appel à 
l'aventure, mais le monologue intérieur et le choix des images suggèrent en contrepoint qu'un véhicule 4/4 peut aussi offrir le confort d'une berline haut de gamme.

Si le quotidien semble un bon argument pour influencer des individus rationnels et les amener à acheter un véhicule « inhabituel » comme un 4/4, l'inverse est encore bien plus vrai. Dans un monde où les nouvelles automobiles sont conçues sur ordinateur et montées dans des chaînes robotisées, si bien qu'elles se ressemblent toutes, peu ou prou, il est indispensable de créer pour chaque modèle une individualité bien définie, de façon à le différencier des autres.

L'exemple de la Renault Clio est tout à fait typique ${ }^{7}$. Ce publi-film de trente secondes nous transporte devant une gentilhommière du sud de la France, où un aristocrate blasé et sa fille font la sieste dans des chaises longues. Profitant du sommeil apparent de son père, la jeune fille, Carole, s'éclipse discrètement, empruntant une Renault Clio, pour aller rejoindre son amoureux au village voisin. Le père, qui feignait seulement l'assoupissement, ordonne alors au chauffeur impassible d'amener une autre Renault Clio, ce qui lui permet d'exercer une surveillance discrète sur le jeune couple, tout en apportant un bouquet de fleurs à un destinataire que nous imaginons féminin. À son retour, la jeune Carole, ne se doutant de rien, retrouve un père apparemment assoupi, et s'enfonce avec soulagement dans sa chaise longue, feignant elle aussi le sommeil. Ces images idéalisées du sud de la France créent une atmosphère de dépaysement, d'opulence, et d'un bien-être affectif mis en valeur par un brin d'illégitimité. On retrouve les ingrédients traditionnels des publicités françaises pour produits de luxe, alcools ou parfums.

Il s'agit donc clairement ici de présenter la Renault Clio comme un véhicule de luxe, en jouant sur l'image de la France à l'étranger. Voilà qui permet d'allier le rêve d'une réussite sociale et amoureuse à la réalité, et l'image idéalisée d'un moi aristocratique à celle d'un véhicule petit et maniable, se faufilant aisément dans les ruelles d'un vieux village. L'image de l'objet s'efface alors devant celle du sujet, et la démonstration cède le pas à l'évocation.

16 En conclusion, la mimétique existe-t-elle vraiment? Vouloir poser les bases d'une "génétique du culturel», à la suite de Richard Dawkins, est un projet dépassant largement le cadre de cette communication, qui s'est bornée à explorer l'analogie entre gène et mème ${ }^{8}$. Ce parallèle s'est néanmoins révélé fructueux à au moins cinq titres différents. À une capacité similaire de se reproduire identiques à eux-mêmes et de s'adapter à leur milieu, mèmes et gènes allient une tendance commune à la compression de l'information. À côté de la densité de données contenues dans les molécules d'ADN, même les ordinateurs les plus puissants sont dépassés. La cherté de l'espace publicitaire conduit parallèlement les annonceurs à saturer de significations le temps limité qui leur est imparti. En quatrième point, gènes et mèmes partagent la capacité d'être indirectement impliqués dans leur environnement. Un gène n'influence directement que la synthèse de certaines protéines. Mais, par une réaction en chaîne, il conduit quand même à donner des yeux bleus ou des pois ridés. De même, rares sont les publicités qui précipitent les consommateurs dans une frénésie d'achats d'impulsion. Néanmoins, les campagnes publicitaires font généralement monter les chiffres de vente.

17 Le dernier point, mais non le moindre, c'est que ni les gènes ni les mèmes ne peuvent s'ériger en destin'. De même que, grâce au génie génétique, l'humanité peut, chaque jour davantage, refuser la fatalité des gènes, de même rien ne nous empêche de faire œuvre 
critique et de démasquer l'imposture d'une image du moi publicitaire où l'être se définirait strictement en fonction d'un avoir mieux ou d'un avoir plus.

\section{BIBLIOGRAPHIE}

Dawkins, Richard. 1976. The Selfish Gene. Oxford : Oxford University Press

\section{ANNEXES}

\section{Annexe 1 : NISSAN}

\begin{tabular}{|l|l|}
\hline Script & Camera Shots \\
\hline $\begin{array}{l}\text { Surely you can't have a special edition with a 1.4 } \\
\text { metre 16 valve engine, electric windows and sun- } \\
\text { roof all for 9265 on the road? }\end{array}$ & $\begin{array}{l}\text { No background. The camera slowly } \\
\text { cooms in on the profile of two Nissan } \\
\text { Both cars then rotate, showing their } \\
\text { Younet. }\end{array}$ \\
$\begin{array}{l}\text { The sunny Flamenco range. See us now, or call 0345 a Nissan. } \\
800600 \text { for more information. }\end{array}$ & $\begin{array}{l}\text { Close up on the Nissan logo. } \\
\text { Long shots of one, then two Nissan } \\
\text { models, alternating with closeups on the } \\
\text { word "Flamenco". }\end{array}$ \\
\hline
\end{tabular}

Annexe 2 : FORD MONDEO

\begin{tabular}{|l|l|}
\hline Script & Camera Shots \\
\hline
\end{tabular}




\begin{tabular}{|c|c|}
\hline $\begin{array}{l}\text { You need strength to protect } \\
\text { yourself. } \\
\text { So every Mondeo has a rigid steel } \\
\text { shell and side-impact bars. } \\
\text { And you want to be safer. } \\
\text { So every Mondeo has a steering- } \\
\text { wheel airbag, seat-belt pre- } \\
\text { tensioners and grabbers, and anti- } \\
\text { submarine seats. Beauty with inner } \\
\text { strength. Mondeo from Ford. }\end{array}$ & $\begin{array}{l}\text { The camera tracks a cloudy sky sideways. } \\
\text { After a medium long shot of the rigid steel shell of a Ford } \\
\text { Mondeo driving against the background of a cliff, the } \\
\text { camera tracks the cloudy sky sideways again, before } \\
\text { closing up first on the rigid steel shell, then on a volcano } \\
\text { crater and a geyser. } \\
\text { The camera pans left to follow a black Ford Mondeo } \\
\text { veering in a clearing surrounded by fir-trees. } \\
\text { Close up on an inflating steering-wheel airbag, then on } \\
\text { the seat-belt pre- tensioners. } \\
\text { The camera pans left to follow a black Ford Mondeo } \\
\text { driving in the cheering light of the sun-rise, before } \\
\text { zooming out to show the vehicle parked in the middle of a } \\
\text { wild open landscape, and zooming in again to leave us } \\
\text { with a final picture of the Ford Mondeo shining under a } \\
\text { clear pale sky. }\end{array}$ \\
\hline
\end{tabular}

\section{Annexe 3 : FOCUS ON FORD}

\begin{tabular}{|c|c|}
\hline Script & Camera Shots \\
\hline $\begin{array}{l}\text { Man: Welcome to "Focus on Ford". Today, seat-belts } \\
\text { are of course fitted to all cars. But do they all work in } \\
\text { the same way? } \\
\text { Woman: In an accident, conventional seat-belts can } \\
\text { allow a little of the belt to spool out from the reel, but } \\
\text { advanced systems have a grabber which locks up the } \\
\text { belt almost instantaneously. } \\
\text { Man: They also have a pre-tensioner to tighten the } \\
\text { diagonal and lap part of the belt, holding you more } \\
\text { securely, and specially designed anti-submarine seats, } \\
\text { which cut the risk of sliding under the belt. } \\
\text { Woman: In a serious collision, these features work } \\
\text { with a steering-wheel airbag, to help reduce the risk } \\
\text { of head and chest injuries. } \\
\text { Man: As part of Ford's dynamic safety engineering, } \\
\text { this system is already standard on every single } \\
\text { Mondeo, and on all new Ford cars for } 1994 \text {. } \\
\text { Woman: That's Ford's commitment to your safety. } \\
\text { Until next time, safe motoring. }\end{array}$ & $\begin{array}{l}\text { Medium close-up of a well-dressed male } \\
\text { presenter walking along the side of a } \\
\text { brand-new black Ford Mondeo in a Ford } \\
\text { demonstration room. } \\
\text { The camera pans left towards an } \\
\text { equally fashionable female presenter } \\
\text { standing on the opposite side of the } \\
\text { Ford Mondeo. } \\
\text { Big close up on the grabber } \\
\text { Close up on the safety-belt } \\
\text { Long shot of an anti-submarine seat } \\
\text { standing on its own without any } \\
\text { background. } \\
\text { Big close-up of a dummy's head } \\
\text { collapsing against a steering-wheel } \\
\text { airbag. } \\
\text { Medium long shot of the male } \\
\text { presenter. The camera tracks out to a } \\
\text { very long shot of the car and the couple } \\
\text { of presenters. }\end{array}$ \\
\hline
\end{tabular}

Annexe 4 : VAUXHALL ASTRA 


\begin{tabular}{|c|c|}
\hline Script & Camera Shots \\
\hline $\begin{array}{l}\text { J: Ah! There you are. } \\
\text { G: It's the Astra specials } \\
\text { demo, J. I'm afraid they } \\
\text { haven't delivered a dummy. } \\
\text { J: What! How can I proceed } \\
\text { without a dummy? } \\
\text { A:J! G! Sorry I'm late. } \\
\text { J: Not only do these Astra } \\
\text { specials include sun-roof, } \\
\text { body-coloured mirrors and } \\
\text { tinted glass, they also boast } \\
\text { a full-size driver's airbag. } \\
\text { Observe! } \\
\text { Atkins, it's well done. } \\
\text { Atkins! Atkins! } \\
\text { A: Only kidding, T. } \\
\text { The astra specials. } \\
\text { California diamonds's } \\
\text { aeroclub. }\end{array}$ & $\begin{array}{l}\text { Long shot of a big demonstration hall in the Vauxhall factory. A } \\
\text { black suited presenter is standing in front of a bright yellow } \\
\text { Vauxhall Astra. } \\
\text { Enters a technician in a white blouse. } \\
\text { The camera zooms in on the men's faces. } \\
\text { A human dummy, looking slightly incongruous in a grey suit, } \\
\text { comes in running, waving "hello" at the same time. Close up on } \\
\text { relief on the presenter's face. } \\
\text { While the technician is fastening the dummy to his seat, the } \\
\text { camera pans right to follow the presenter walking along a row of } \\
\text { Vauxhall models. The camera tilts up to show a crowd of } \\
\text { reporters watching from a glass cage upstairs. } \\
\text { As the presenter points to the car, the Vauxhall model starts } \\
\text { apparently on its own, gains speed, and crashes against a metal } \\
\text { obstacle. Medium close-up on the inflating air bag. The dummy } \\
\text { winks at us. The presenter grins in relief looking up at the } \\
\text { presenters clapping upstairs, then quickly walks towards the car } \\
\text { and claps on the dummy's shoulders, then shakes him as there is } \\
\text { no response. } \\
\text { The dummy then sits up, laughing. The presenter shakes his fist } \\
\text { at him, looking up quickly to check that the reporters haven't } \\
\text { noticed anything. } \\
\text { Long shot on four motionless Vauxhall Astra models set in a } \\
\text { square, over the words: "Vauxhall. For more information call } 0888 \\
444200 \text { " }\end{array}$ \\
\hline
\end{tabular}

\section{Annexe 5 : LAND ROVER (man's voice)}

\begin{tabular}{|l|l}
\hline Script & Camera Shots
\end{tabular}




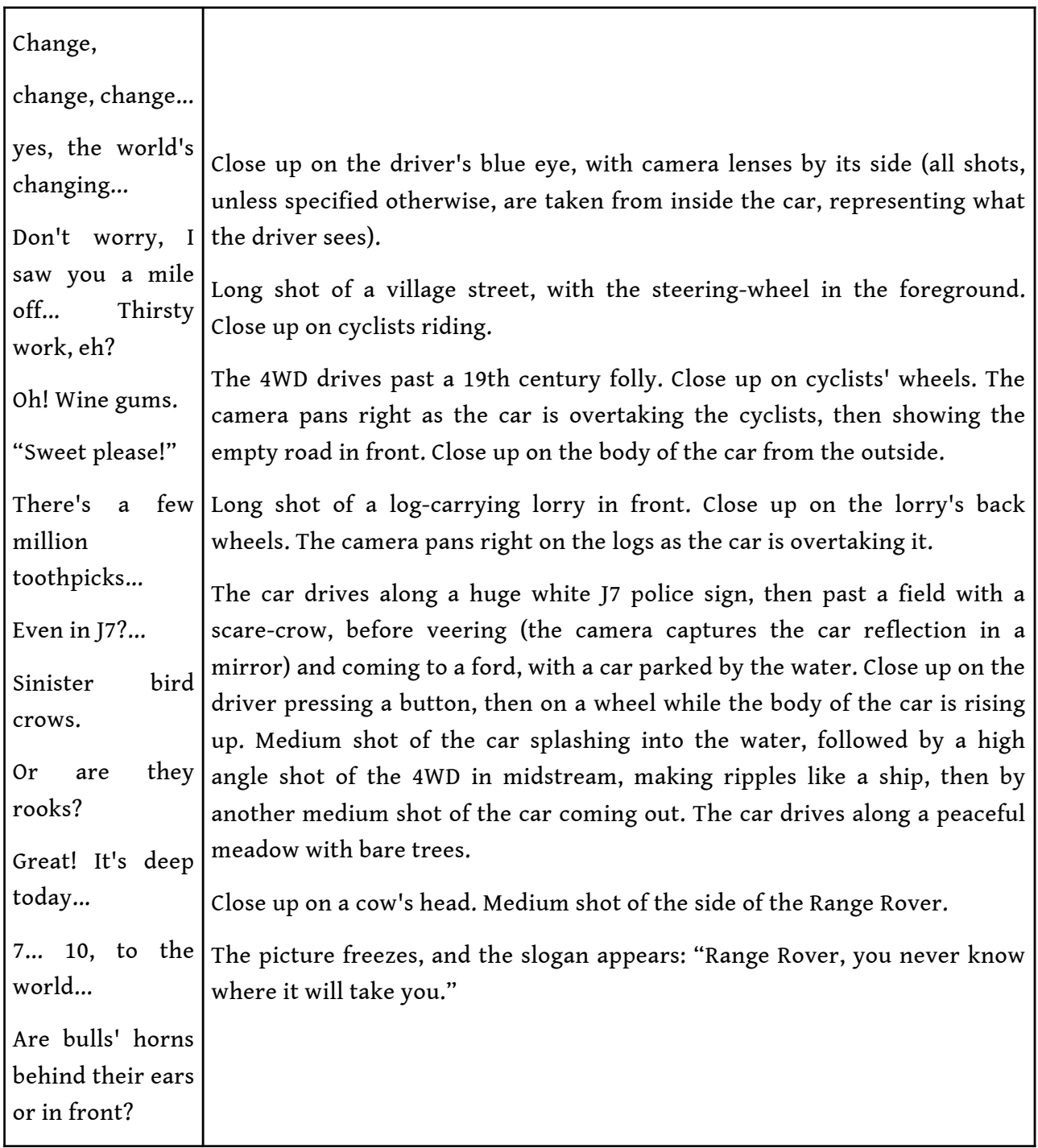

\section{Annexe 6 : LAND ROVER (woman's voice)}

\begin{tabular}{|l|l}
\hline Script & Camera Shots
\end{tabular}




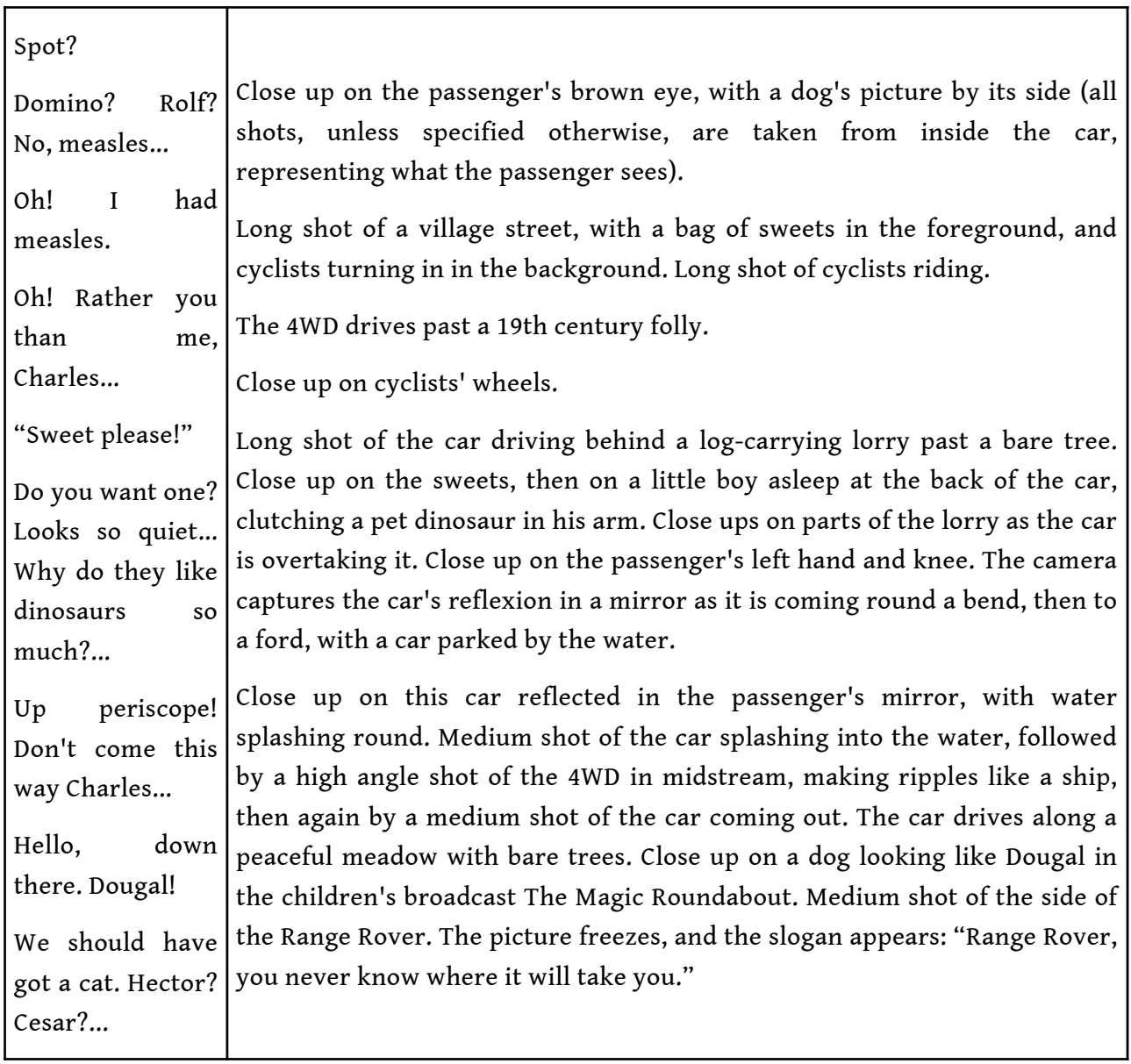

\section{Annexe 7 : RENAULT CLIO}

\begin{tabular}{|l|l}
\hline Script & Camera Shots \\
\hline
\end{tabular}




\begin{tabular}{|c|c|}
\hline $\begin{array}{l}\text { "Papa?" } \\
\text { You may be } \\
\text { looking for a car } \\
\text { that's small and } \\
\text { practical, but you } \\
\text { still want a car } \\
\text { that feels } \\
\text { luxurious. } \\
\text { Well, now you've } \\
\text { found it. Because } \\
\text { while the clio is } \\
\text { certainly small, } \\
\text { it's perfectly } \\
\text { forward. } \\
\text { "Carole?" } \\
\text { "Papa?" } \\
\text { "The Renault } \\
\text { Clio." }\end{array}$ & $\begin{array}{l}\text { Close up on a young lady in a summer frock, standing up quietly from a } \\
\text { wicker armchair. Panoramic shot of her father having a nap in front of a } \\
\text { mansion in the South of France. } \\
\text { Taking advantage of the fact that her father looks fast asleep, Carole takes a } \\
\text { Renault Clio parked under a thick stone vault not far away from aristocratic } \\
\text { old black Citroen limousines, and drives off. The impassive chauffeur in } \\
\text { uniform can't help a smile. Close up on Carole's finger pressing a button. } \\
\text { The father opens an eye at the noise of the Clio driving away along a } \\
\text { narrow road lined with plane trees to the neighbouring village, where her } \\
\text { boy friend greets her in style, opening the door for her. Medium shot on the } \\
\text { father, beckoning the chauffeur who comes along driving another Renault } \\
\text { Clio. Medium close up on the young couple, Carole's boy friend twirling her } \\
\text { round joyfully. } \\
\text { The father drives through the narrow streets of the village, past the young } \\
\text { couple sitting outside a café, then stops in front of an aristocratic old house } \\
\text { adorned with a climbing vine. The father rushes up the steps, holding a } \\
\text { bunch of flowers. An attractive young lady comes out to greet him. } \\
\text { Medium shot on Carole slipping back in her chair. Her father, sitting in the } \\
\text { very same position where she left him, as if nothing had happened, opens } \\
\text { an eye and pretends to be disturbed in his sleep. } \\
\text { Long shot of both clios parked by the door. A notice appears: Ask about } \\
\text { your Renault diamond deal now. }\end{array}$ \\
\hline
\end{tabular}

\section{NOTES}

1. Cf. annexe 1.

2. Cf. annexe 2.

3. "you need strength", "you want to be safer", devient "seat belts are of course fitted to all cars", "advanced systems have a grabber ", « these features work with a steering-wheel airbag ", etc.

4. Cf. annexe 2 .

5. Cf. annexe 3.

6. "How readily our thoughts swarm upon a new object, lifting it a little way, as ants carry a blade of straw so feverishly, and then leave it... " remarque la narratrice de la nouvelle " The mark on the wall », et cette phrase pourrait fort bien servir d'exergue aux deux publicités pour Rover (Virginia Woolf, 1962, «The mark on the wall», In Norton Anthology of English Literature, vol. 2. New York : Norton, 2309).

7. Cf. annexe 7.

8. Le rapport entre mimétique et sémiotique n'a pas davantage pu être développé dans cette communication, pour des raisons similaires. Voici quelques jalons de comparaison cependant. Sèmes et gènes ont des caractéristiques communes : il s'agit dans les deux cas d'unités minimales d'information, qui peuvent se combiner (en chromosomes ou en signes). Ils se définissent néanmoins selon des critères différents. Alors que la définition d'un gène est fonctionnelle (c'est le fragment de l'ADN du petit pois qui lui donne, par exemple, une texture lisse), la définition d'un sème est relationnelle (ce qui différencie un pois gourmand d'un petit pois ordinaire, par exemple, c'est le sème « gousse comestible »).

9. Cf. Richard Dawkins (1976: 121) : «We alone, on earth, can rebel against the tyranny of the selfish replicators $»$. 


\section{RÉSUMÉS}

Le thème des voitures a été sélectionné parce qu'il est au carrefour entre caractéristiques techniques et motivations d'achat d'ordre culturel (rêves, images de soi, valeurs). Le concept de mème, forgé par le zoologiste britannique Richard Dawkins pour transposer dans le domaine culturel la notion de gène, permet d'analyser comment les publicités de voitures sont sujettes à des mutations à partir d'un " gène culturel ", ou mème originel. Dans le mème originel, le texte se borne à décliner, et les images à illustrer les caractéristiques techniques de tel ou tel modèle. À partir de là, on peut repérer une première mutation formelle, qui joue sur le changement de type de discours, depuis le sérieux du documentaire scientifique jusqu'au burlesque de la farce. On peut ensuite distinguer une deuxième mutation, où l'accent n'est plus mis sur le produit (la voiture), mais sur le destinataire (l'acheteur potentiel). L'information cède alors le pas à la signification.

The example of car advertisements has been selected because they contain both technical characteristics and a cultural dimension (through dreams, self image, values). The concept of meme, coined by the zoologist Richard Dawkins to extrapolate the findings of genetics to human culture, enables us first to define a basic meme in car advertisements, and then to explore its various mutations. In the basic meme, the text, illustrated by the pictures, describes the technical characteristics of a particular car. The first mutation is on form only, and plays on a change in the type of discourse, from the serious style of the popular science broadcast to parody and farce. In the second mutation, the focus shifts from the product to its potential buyer. Information then gives way to meaning.

\section{INDEX}

Mots-clés : discours, publicité

Keywords : advertising, discourse

\section{AUTEUR}

\section{MARIE-HÉLÈNE FRIES-VERDEIL}

Marie-Hélène Fries-Verdeil enseigne à l'Université Joseph-Fourier-Grenoble 1. Marie-

Helene.Fries@ujf-grenoble.fr 